\title{
Lung ultrasound: a valuable tool for the assessment of dialysis patients with COVID-19
}

\author{
Nathaniel Reisinger ${ }^{1} \cdot$ Abhilash Koratala $^{2}$
}

Received: 8 April 2020 / Accepted: 10 May 2020 / Published online: 19 May 2020

(c) Japanese Society of Nephrology 2020

\section{To the Editor,}

Patients on maintenance hemodialysis face unique challenges during Coronavirus disease 2019 (COVID-19) pandemic due to frequent contact with health care facilities as well as high comorbidity burden subjecting them to increased risk of serious complications from the disease. With an ongoing surge in COVID-related hospitalizations exhausting resources, outpatient dialysis units are preparing to manage patients suspected to have or tested positive for COVID-19 with mild symptoms. In addition to exploring the options to safely dialyze these patients, timely recognition of clinical deterioration and referral to the hospital are crucial [1]. Symptom recognition is not straightforward as these patients often have multiple etiologies for dyspnea especially given concomitant pulmonary congestion due to fluid overload. In some of the severely affected parts of the world, triage protocols have been developed using computed tomography (CT) scan of the chest as the primary imaging modality to identify dialysis patients that need hospitalization. Repeated studies were often performed in those with positive findings, exposing patients to significant ionizing radiation [2].

In contrast, lung ultrasound (LUS) is a bedside diagnostic tool that emits no ionizing radiation and has comparable diagnostic accuracy to that of CT scan in detecting most lung pathologies. Moreover, LUS has a proven track record

Electronic supplementary material The online version of this article (https://doi.org/10.1007/s10157-020-01903-x) contains supplementary material, which is available to authorized users.

Abhilash Koratala

akoratala@mcw.edu

1 University of Pennsylvania Health System, Philadelphia, PA, USA

2 Division of Nephrology, Room A 7633, Medical College of Wisconsin, $8701 \mathrm{~W}$ Watertown Plank Rd, Wauwatosa, WI 53226, USA in dialysis patients to assess extravascular lung water, and nephrologists at some centers use it routinely for this purpose [3]. Lung imaging findings of COVID-19 are non-specific on both CT scan and LUS and cannot differentiate among viral pneumonias. Preliminary data suggest that the sonographic features of this disease correlate well with chest CT features. In fact, the detection of focal B-lines on LUS may be more sensitive than CT findings in early disease [4]. Other characteristic features of COVID-19 disease on LUS include a thickened or irregular pleural line, subpleural consolidations, and multifocal or confluent B-lines. Translobar consolidation and pleural effusions are relatively uncommon [5].

We herein present a brief case study to illustrate the role of LUS in this context. A 79-year-old woman with a history of end-stage kidney disease on maintenance hemodialysis and hypertension was found to be hypoxemic with an oxygen saturation of $90 \%$ on room air. Bedside LUS demonstrated patchy areas of pleural thickening and irregularity as well as confluent B-lines and scattered consolidations consistent with viral pneumonia (Fig. 1, supplemental video 1). Focused cardiac ultrasound did not reveal any overt signs of fluid overload. She was tested positive for COVID-19 and subsequently improved with supportive care. The patient continued to receive dialysis during a separate shift dedicated to COVID-19-positive patients.

From the standpoint of infection control, using LUS instead of CT limits staff exposure to the virus during transportation and avoids the downtime for radiology room decontamination, which takes about an hour after each scan. The key advantages of LUS in this setting are summarized in Fig. 2. We propose that dialysis units adopt LUS as a bedside tool to diagnose and monitor the extent of pulmonary involvement in patients with COVID-19 and differentiate from other causes of dyspnea. With the availability of cheaper ultra-portable hand-held ultrasound devices, LUS is even more practical. CT scan can be reserved for those with equivocal LUS findings or underlying chronic lung disease that interferes with interpretation. 

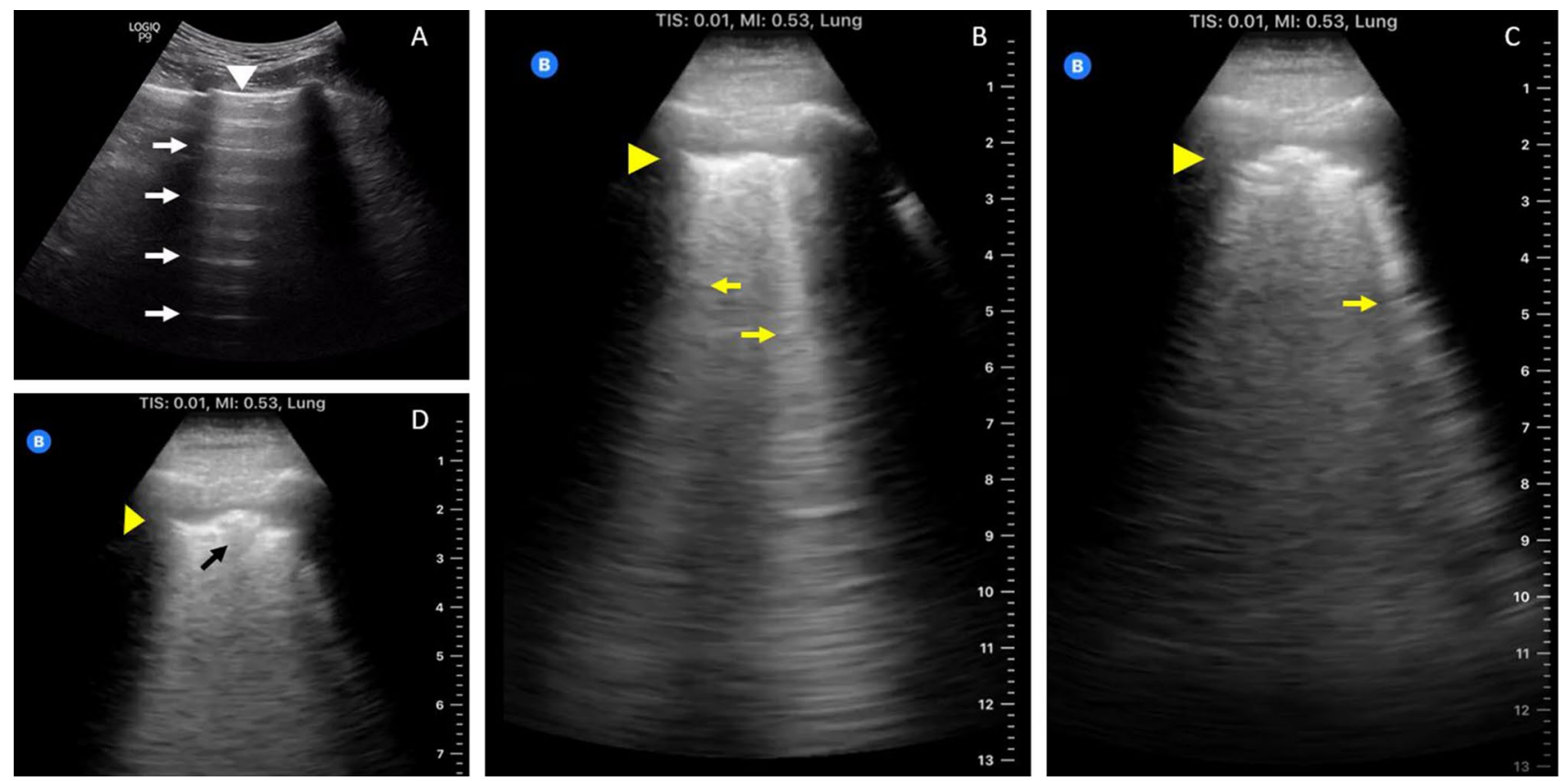

Fig. 1 a Normal lung ultrasound demonstrating horizontal A-lines (arrows). Arrowhead indicates normal pleural line. b-d Lung ultrasound images obtained from a COVID-19-positive dialysis patient using hand-held Butterfly ${ }^{\circledR}$ ultrasound device: arrowhead points to the pleural line demonstrating thickening and irregularity. Black arrow points to a subpleural consolidation. Yellow arrows point to vertical B-lines that replace A-lines. Note that these B-lines are confluentalmost merged giving a 'white lung' appearance

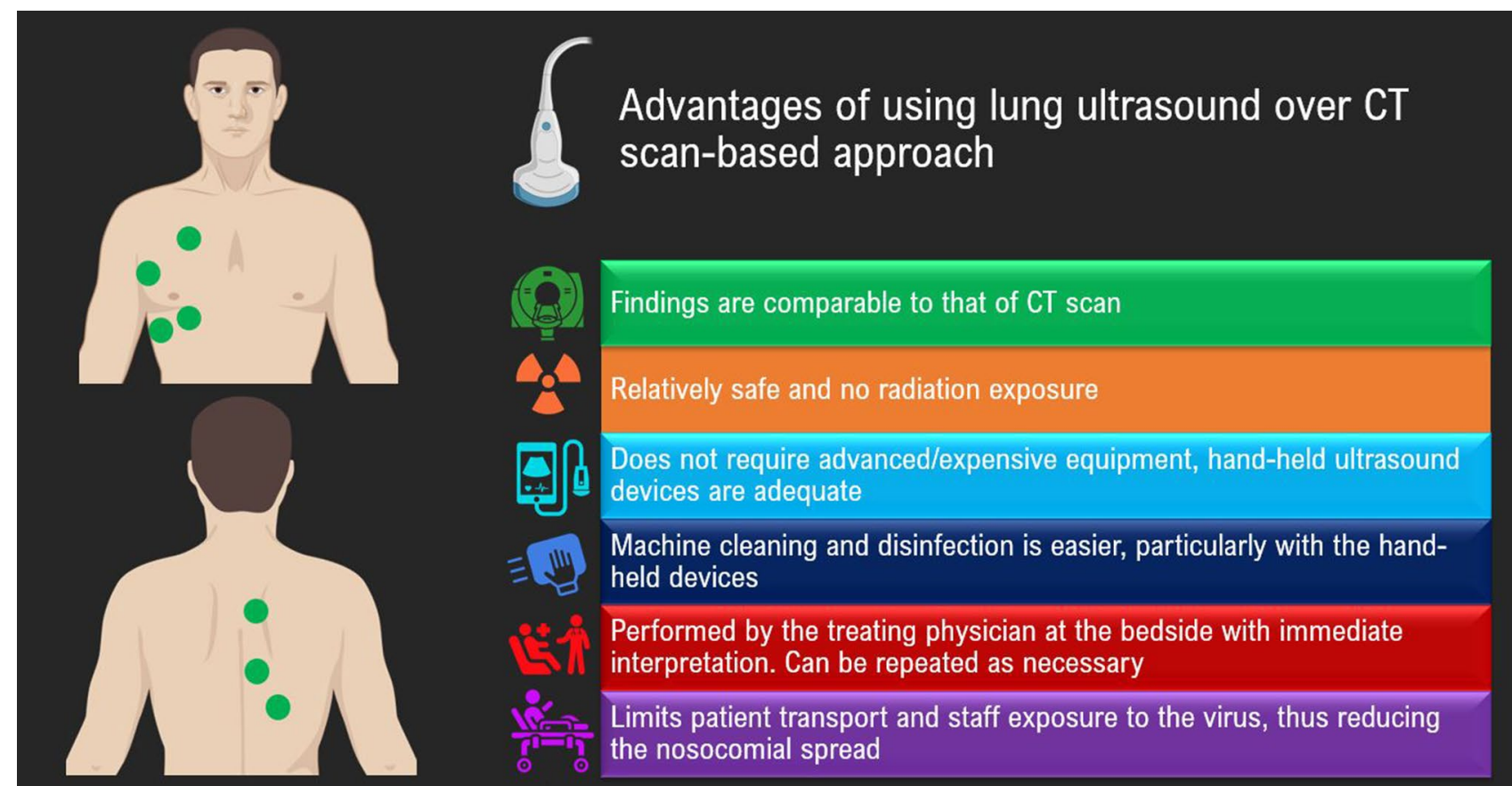

Typical chest scan areas

Fig. 2 Advantages of lung ultrasound compared to CT scan in managing patients with COVID-19. Left panel illustrates the typical ultrasound scanning sites used to detect the lung lesions (anterior, lateral, and posterior) 
Right now, dialysis units lack trained staff for performing and interpreting LUS. However, most medical professionals can be taught to perform 'goal-directed' LUS quickly. Web-based and remote-mentored training protocols demonstrate high inter-observer agreement for pathologic findings after a short training period [6]. Adding to this, numerous free resources exist for online training and remote mentoring. Further, cloud-based image archiving platforms allow for immediate remote quality assurance easier with expert physicians reviewing images generated by novice users remotely. The time is now to act to prepare for the crisis and provide best possible care to our patients.

Author contributions Both the authors have made substantial contribution to the preparation of this manuscript.

Funding None.

\section{Compliance with ethical standards}

Conflict of interest The authors have declared that no conflict of interest exists.

Human and animal rights (with IRB approval number) This article does not contain any studies with human participants or animals performed by any of the authors.

Permissions Human body images in Fig. 2 were created using BioRender $^{\circledR}$.

\section{References}

1. Kliger AS, Silberzweig J. Mitigating risk of COVID-19 in dialysis facilities. Clin J Am Soc Nephrol. 2020;15(5):707-9. https://doi. org/10.2215/CJN.03340320.

2. Li J, Xu G. Lessons from the experience in Wuhan to reduce risk of COVID-19 infection in patients undergoing long-term hemodialysis. Clin J Am Soc Nephrol. 2020;15(5):717-9. https://doi. org/10.2215/CJN.03420320.

3. Koratala A, Ronco C, Kazory A. The promising role of lung ultrasound in assessment of volume status for patients receiving maintenance renal replacement therapy. Blood Purif. 2020. https ://doi.org/10.1159/000505529.

4. Peng QY, Wang XT, Zhang LN, Chinese Critical Care Ultrasound Study Group (CCUSG). Findings of lung ultrasonography of novel corona virus pneumonia during the 2019-2020 epidemic. Intensive Care Med. 2020. https://doi.org/10.1007/s00134-02005996-6.

5. Huang Y, Wang S, Liu Y, Zhang Y, et al. A preliminary study on the ultrasonic manifestations of peripulmonary lesions of noncritical novel coronavirus pneumonia (COVID-19). 2020. PREPRINT (Version 1) available at Research Square: https://www. researchsquare.com/article/rs-14928/v1. Accessed 4 June 2020.

6. Gargani L, Sicari R, Raciti M, Serasini L, et al. Efficacy of a remote web-based lung ultrasound training for nephrologists and cardiologists: a LUST trial sub-project. Nephrol Dial Transplant. 2016;31(12):1982-8.

Publisher's Note Springer Nature remains neutral with regard to jurisdictional claims in published maps and institutional affiliations. 